\title{
23
}

\section{Soft Systems Methodology - A Process View}

\author{
Graham Tate \\ Information Systems Department, City Polytechnic of Hong Kong \\ 83 Tat Chee Avenue, Kowloon, HONG KONG
}

\begin{abstract}
Methods from the relatively recent research area of software process modelling are applicable to more than just software processes and methodologies. In this study, software process modelling is applied to the Checkland/Wilson soft systems methodology (SSM). The primary objective is a better understanding of SSM. Advantages and disadvantages of doing so are identified, including the possible disadvantage of over constraining SSM and impairing its spontaneity. A small set of requirements is given for the process modelling and enaction of an instance of SSM. These include low intrusiveness and high practitioner freedom within a basic set of specified activities and products. Goals of modelling and enaction include the observation and better understanding of SSM in action, the better definition of it in situations where this is appropriate, the identification of areas of difficulty for the practitioner(s) involved, the measurement of effort on activities, including iteration and rework, and the possible provision of guidance to inexperienced practitioners. A prototype model is described based on these requirements. Some preliminary findings based on the enaction of a prototype process model in an SSM case study are described.
\end{abstract}

Keyword Codes: D.2, D.2.1, D.2.9, D.2.m

Keywords: Software Engineering; Requirements/Specifications; Management; Miscellaneous

\section{INTRODUCTION}

Our primary objective is the application of software process modelling to soft systems methodology (when used for determining information systems requirements) for the purpose of observing SSM in action and understanding it better.

The first and most difficult problem in many information systems (IS) situations is determining requirements. It is also the first essential step in the production of quality systems. No matter how high the quality of information systems is in other respects, if the requirements are wrong, the system will be wrong.

Soft Systems Methodology (SSM) is a purposeful, but relatively unconstrained approach to the determination of requirements for an information system (IS), or indeed for any system. In an IS context it aims to identify the essential information needs of a business operation without too much noise or interference arising from consideration of the way things are done at present. This is in sharp contrast to more traditional methods that examine the current operation in detail in an attempt to determine where changes should be made. One of the more systematic formulations of the latter, the 'change analysis' approach, is given in Lundeberg et al ${ }^{1}$. SSM attempts to take an 'ideal world' view, concentrating on essence rather than accidents, before tempering it with the constraints of the real world.

SSM is intentionally 'soft' in that it addresses unstructured problem situations and tries not to be too dogmatic about how its practitioners go about finding solutions to them. Indeed, there are probably as many variants of SSM as there are active practitioners. We shall therefore concentrate on the fundamentals of SSM as expressed in 7 step Checkland methodology that is the basis of later approaches that claim to be $\mathrm{SSM}^{2,3}$. The first six steps are:

1 Investigate the problem situation. 
2 Express the problem situation in some suitable form(s).

3 Formulate root definitions of relevant systems.

4 Build conceptual models.

5 Validate the conceptual models against the problem situation expression.

6 Define feasible desirable changes to the existing situation.

The seventh step involves action to solve the problem or improve the situation. If we regard SSM as primarily a requirements methodology, then step seven is concerned with implementation, which we will not consider further here.

This paper is concerned with the application of process modelling to SSM. Process modelling in this context means using an appropriate modelling notation to define the SSM process at a suitable level of detail, including the activities and products of interest. Furthermore it means executing, or in process modelling terms, enacting the defined process, keeping a record of the enaction, modifying the process model if and when necessary to correspond with what is actually done, and measuring aspects of the process, if practicable.

Some care is taken in establishing requirements for SSM modelling, since process models can differ greatly according to their purposes. A modelling architecture is described together with a low-intrusion, large-grain, exploratory process model for SSM. Experience with the use of the model in a case study by a relatively inexperienced SSM practitioner is described. As a result of this exploratory study some preliminary conclusions are drawn about the feasibility and usefulness of process modelling for SSM.

\section{WHAT IS A PROCESS VIEW?}

Taking a process view of SSM means focusing on the SSM process itself, its activities, products, events, milestones, etc., and their relationships to each other. It involves building one or more models of the process in order to understand, define and enact it. Enaction of a process model is like execution, but with much of the work (in this case almost all the work) being done by the practitioner(s) concerned. If a higher level of computer assistance of the process is used, via a workbench for example, then enaction becomes a sort of symbiosis of practitioner, workbench and model.

A particular process view depends on the viewer, the viewpoint, the goals, purposes and desired outputs of the modelling exercise and the level of definition, e.g. microscopic or macroscopic.

Though process modelling has been done informally for as long as men have used complex processes, the emergence as a major research field of process modelling for software, and software related processes such as SSM, is relatively recent. A major milestone in process modelling was the 1987 paper of Leon Osterweil "Software processes are software too"4. As the title indicates, the modelling can be rather formal and detailed, analogous to the manner in which a computer program models a computational or logical process.

\subsection{Process modelling}

A survey of process modelling is given in Figure 1 , taken from Madhavji ${ }^{5}$. In relation to SSM, process engineering is the modelling of the particular SSM process that we have chosen to use. This could be a convenient instantiation of a generic SSM process. Process performance is the enaction of the chosen process by practitioner(s), i.e. the performance of an actual SSM study, including the production of conceptual models and recommendations for feasible desirable changes. Sector B of the diagram relates to the management of the process. This may be minimal for many single practitioner SSM studies, but is obviously important for larger studies and for other more protracted processes, such as software development.

There are a number of process modelling methods and notations. These include:

- process programming in which a process is specified using programming language notation, such as Ada PDL (program design language)

- entity-process modelling in which the states of process objects of interest are modelled together with the events which cause transitions between them 6

- special process modelling languages and notations, or extensions to existing languages, for example those used in the ambitious Arcadia project 7 
- more conventional IS modelling - after all processes are systems - using for example one of the many structured analysis and design or object-oriented analysis and design notations.

Most of these process modelling methods can be used in most modelling situations, though in any particular situation some will be better than others. Most applications have been to more detailed software processes but the methods are generally applicable, with greater or less convenience, to the modelling of any definable process at any appropriate level of detail, using either fine grain models, or coarse grain models.

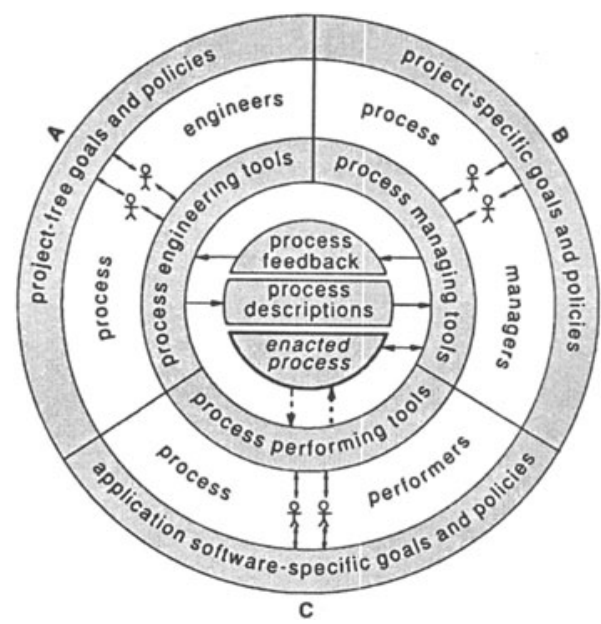

Figure 1: Madhavji's process modelling model

2.2 Process modelling \& enaction in practice

The process modelling literature has a great deal to say about how modelling can or should be done, but very little about actual experience of doing it. The requirements for process modelling in practice are seldom made explicit. They can include:

understanding the process

process definition, e.g. for standardisation

measurement and data collection during process enaction

collecting design $\&$ decision history during process enaction

guidance of practitioners

control of the process and those performing it

management, including risk management.

Clearly, the nature, granularity, and intrusiveness of a process model will depend on the requirements it has to meet. The modelling notation and tools used in any particular case will depend on requirements and tool availability. 


\section{WHY TURN A PROCESS SPOTLIGHT ON SSM?}

The initial reasons for doing so include a better understanding of the process and explicit observation of the process in practice leading to basic recording, measurement \& data collection. Other reasons, which are a little further down the track, include: the identification of common variations of SSM (perhaps different instantiations of the of the same essential generic process); the guidance of practitioners, particularly inexperienced practitioners through, for example the provision of checklists; risk management, covering possible risks in the SSM process itself and also in its products; the identification of needs for tools, e.g. an SSM dictionary of repository. Other possible SSM process modelling advantages include: ensuring that no essential processes are omitted or skimped; the identification of areas of uncertainty, difficulty, high effort, frequent iteration and rework; the possible provision of some problem situation-solution traceability.

There are also a number of possible disadvantages of SSM process modelling which must be borne in mind. The enaction of an SSM process model may unduly interfere with the SSM process itself. This is a potential problem with the enaction of any process model. A balance is necessary between guidance and interference. The process model may over-define the process, over-constrain its enaction and not allow for essential variations, iterations, etc. Enaction may inhibit creativity, spontaneity and enthusiasm, which many practitioners would say are essential elements of SSM. Enaction may also distract from the main aims of SSM, though a good process model should emphasise these.

A further reason for modelling SSM is the future potential for monitoring aspects of the quality the SSM process, particularly in situations where there are a number of practitioners with different backgrounds and experience.

\section{REQUIREMENTS FOR AN INITIAL SSM PROCESS MODEL}

Where model building is concerned, the importance of determining requirements can scarcely be overemphasised. In spite of the substantial effort required to build and use process models, requirements determination seems either to have been largely overlooked or to have not been explicitly reported in the literature. In the case of SSM, however, where the methodology being modelled is not as well-defined as most others that have been modelled, the question of requirements cannot be brushed aside quite so easily. In brief, our key requirements for an initial SSM process model are those summarised below.

Only key activities and products will be modelled. These are the production of the key products, namely problem situation expression, root definitions, conceptual models, consensus conceptual model, conceptual model validation notes, feasible desirable change report. Subsidiary activities will be subsumed under these major activities. If there are difficulties in doing this, then these will be noted in the enaction records. Flexible sequencing of activities will be permitted to allow for iteration, rework, out of sequence activities, etc. The granularity of the model should be large, on the human time and effort scale rather than the computer scale. The aim is minimal intrusiveness consistent with observing the SSM process and collecting data (such as time, effort and product data) about it. Other requirements include the ability to include useful checklists for practitioner help and guidance, if needed, and interfaces to permit existing or planned tools to be accommodated conveniently within model enaction. Essentially we are seeking to build a management-level SSM shell to support observation, data collection, measurement, tool use, help and possible guidance.

\section{APPROACHES TO MODELLING SSM}

Figure 2 shows the SSM process modelling architecture used. It will be noted that the SSMer (or SSM practitioner) interacts with the SSM enaction engine, which aims to be an assistant and recorder of all on- \& off-workbench SSM activity (or as much as can be easily captured, automatically or in userfriendly exchanges). The SSM process model is essentially a plug-in specification which is interpreted by the enaction engine. Any tools are accessed through enaction so that time spent on tool use can be measured as well as product change (e.g. in size or complexity) as a result of tool use. The design of the SSM enaction engine itself is similar to that described by Tate ${ }^{8}$. 


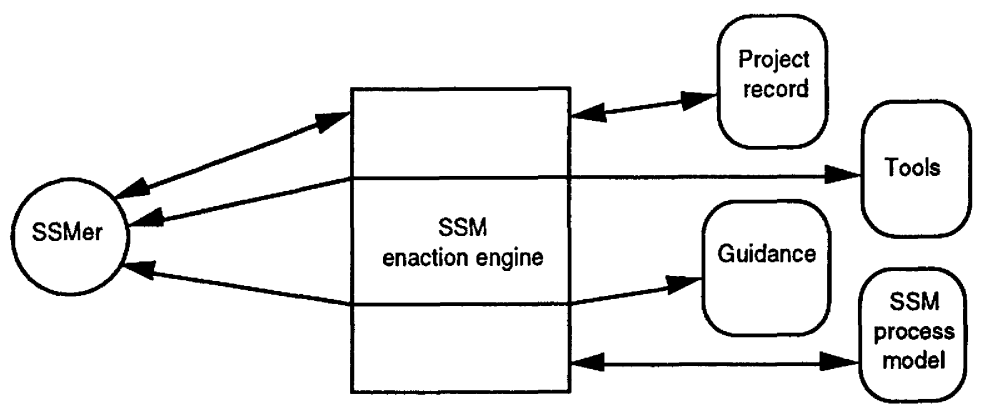

Figure 2: SSM process modelling architecture

Such a workbench-based architecture obviously only covers on-workbench activities directly. Information about off-workbench activities can be collected in several ways. It may be entered during the next on-workbench session. This is the solution we have currently adopted. In future personal digital advisors (PDAs) may be used to help with the incorporation of off-workbench activity into the enaction records.

5.1 A low-intrusion generic model

The initial model used is an instantiation of a simple generic product-based model. The building block of the model is shown in Figure 3. One such block is included for each of the six key SSM products listed in the requirements. Though there are no data flows connecting the blocks, they are dependent on each other through the product base to which they all contribute. No sequence constraints on steps are imposed other than data availability. The SSMer determines the sequence of activities, thus allowing for any pattern of work or rework. Initially we just record simple data and measurements such as the product worked on, what was done and why, when it was done, the effort expended, the product size (if available) at end of the session (e.g. text page or word count, diagram page or object count), and any unresolved problems and consequent actions arising from session.

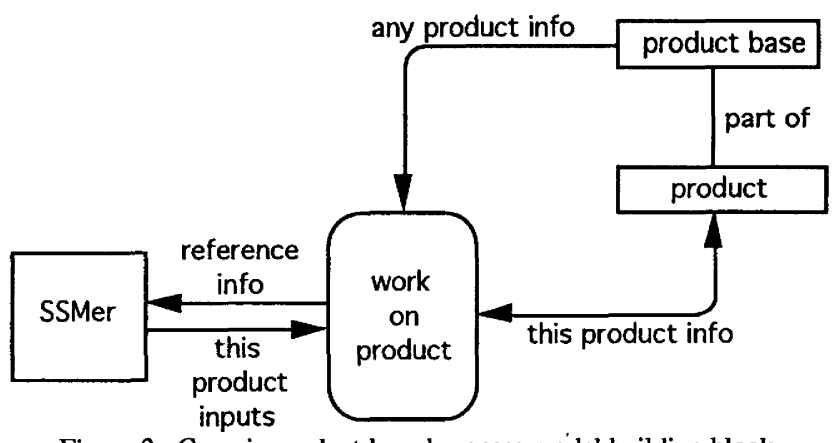

\section{THE CASE STUDY}

Figure 3: Generic product-based process model building block

The case study itself is not important to this investigation save as a vehicle for the enaction of a prototype of the simple SSM process model outlined above. The case study had to do with the requirements for an information system to support the work of IS journal editors. This may seem a simple and well-defined problem, perhaps even a 'hard' problem in SSM terminology. However, if one considers questions such as special issues, departments, markets, interest and readability, the vagaries of 
the traditional refereeing process, very limited editorial time and other resources, and so on, the problem becomes somewhat soggy and the views of the main actors rather divergent.

We walked through an enaction of the case study using the process model described. Workbench support was rudimentary, mainly consisting of menu selection of one of the six activities modelled, time recording and the collection of notes and observations about each activity. Products in text form were kept on the workbench, but not at this stage integrated with the enaction engine as the architecture of Figure 2 indicates. Nevertheless, the architecture and model were followed strictly. Other products, such as conceptual models, were paper based, as is traditionally the case in SSM. The findings from our enaction of the case study problem are described below in the conclusions.

\section{CONCLUSIONS}

Process modelling of SSM is feasible. But is it useful? Our conclusions at this stage are, on balance, yes. It helped an inexperienced practitioner to understand what he was trying to do and where each of his SSM activities fitted into the overall process. At each point, it also helped to focus attention firmly on the activity in hand and its product so that the practitioner was fully aware of his position, the current state of the process, and his immediate target. A good handbook or even a informal model can not quite do this, at least not without the user mentally transforming his references to it into implicit process model fragments which may not be complete and will likely require repeated instantiation, not always in the same form.

Did process modelling stifle creativity? Not in this case. The creative work still had to be done. Model enaction was more intrusive than one would have liked, but not too inconvenient. Observation and measurement intrudes into any process, particularly into complex processes like requirements determination or system development.

What did process model enaction reveal? For this (admittedly inexperienced) practitioner, it showed that SSM involves a great deal of iteration. There was a starting point and a finishing point but the activities in between, while exhibiting a general progression from beginning to end, often occurred rather more in parallel with the practitioner moving from product to product refining and improving them more or less together. In this process, the use of the process model, crude as it was, helped materially to avoid what Raymond Oakes, a well-known SSM practitioner in Northern Ireland, has referred to as "reality seepage", namely the undue intrusion of the 'real' world into the 'ideal' world of conceptual models This was particularly important because of the number of times the practitioner's activities crossed the real/ideal boundary, often within a short period of time.

This practitioner also felt a strong need for tools, particularly in the enaction environment. Without tools the human partner in enaction carries the lion's share of the effort and there is much less integration than the same developer has experienced in a CASE environment ${ }^{8}$.

Other conclusions are that SSM process modelling can be done with relatively simple techniques and tools and that it could potentially be a starting point for measuring, better understanding, refining and guiding SSM, particularly for beginners in SSM.

Further experiments are necessary to evaluate the usefulness of SSM modelling and enaction in a wider context.

\section{REFERENCES}

1. Lundeberg M., Goldkuhl G. and Nilsson A. Information Systems Development: A Systematic Approach Prentice-Hall, Englewood Cliffs, NJ, USA (1981)

2. Checkland P.B. Systems Thinking, Systems Practice John Wiley, Chichester, UK (1981)

3. Wilson B. Systems: Concepts, Methodologies and Applications 2ed., John Wiley and Sons Ltd., Chichester, UK (1990)

4. Osterweil L., "Software Processes are Software Too" Proc. 9th ICSE, (Monterey, CA, March 30-April 2 1987), ACM 2-13

5. Madhavji, N. H. "The process cycle" Software Engineering Journal (Sept 1991) 234-242

6. Humphrey, W.S. and Kellner, M.I. "Software Process Modelling: Principles of Entity Process Models" Proc. 1Ith ICSE (Pittsburgh, Pa, May 1989) 331-342

7. Taylor, R. N., Belz, F. C., Clarke, L. A., Osterweil, L., Selby, L., Wileden, J. C., Woll, A. L. and Young, M. "Foundations for the Arcadia environment architecture" in Henderson, P. B. (ed) Proc. 3rd ACM SIGSOFT/SIGPLAN Software Engineering Symposium on Practical Software Development Environments, Boston (Nov 1988) pp 1-13

8. Tate, G. "Software process modelling and metrics: a CASE study" Information and Software Technology Vol 35 No $6 / 7$ (June/July 1993) pp 323-330 